\title{
Intelligent Weight Machines with Load Adjustment Based on Servo Drive (Project Under Development)
}

\author{
Vitaliy V. Episheva, Dmitriy G. Maksakov , \\ Konstantin V. Korovin ${ }^{b}$, Vadim V. Erlikha, \\ Alexey A. Petrova and Lina N. Petrova ${ }^{a *}$ \\ ${ }^{a}$ South Ural State University (National Research University) \\ Chelyabinsk, Russian Federation \\ ${ }^{b}$ Dzhimnes company \\ Chelyabinsk, Russian Federation
}

Received 27.10.2020, received in revised form 05.11.2020, accepted 04.02.2021

\begin{abstract}
The study aims to create a new generation weight machine for obtaining objective information (digital control) about the performed load and the response of the cardiovascular system and the musculoskeletal system during exercise. Patent search, publication analysis, marketing research, and three research and development activities were carried out. Technical requirements for intelligent weight machines were formulated. Based on the Gravitron training machine, 2 prototypes were created, where traditional weight stacks were replaced by a system consisting of a servo drive and control system components. To control the biomechanics of movement, a prototype consisting of a force platform integrated into the seat of the weight machine was developed. Biofeedback is implemented by displaying data on the center of pressure in the initial position and during exercise. A prototype of an ECG monitoring system in standard lead I was created, which is software and hardware built into the bar of a weight machine. The primary results demonstrate the technical feasibility of creating a new class of training machines with biofeedback and continuous monitoring of the user's state. Combining several intelligent machines into a single gym ecosystem will allow developing fundamentally new approaches to the training process.
\end{abstract}

Keywords: weight machine, servo drive, ECG monitoring, control of the center of pressure, intelligent training machine.

This work was accomplished as part of the state assignment of the Ministry of Science and Higher Education of the Russian Federation FENU-2020-0022, No. 2020072.

Research area: theory and methodology of physical education, sports training, healthimproving and adaptive physical culture.

(C) Siberian Federal University. All rights reserved

* Corresponding author E-mail address: epishevvv@susu.ru ORCID: 0000-0002-7284-7388 (Epishev) 
Citation: Epishev, V.V., Maksakov, D.G., Korovin, K.V., Erlikh, V.V., Petrov, A.A., Petrova, L.N. (2021). Intelligent weight machines with load adjustment based on servo drive (project under Development). J. Sib. Fed. Univ. Humanit. Soc. Sci., 14(2), 241-249. DOI: 10.17516/1997-1370-0715.

\title{
Интеллектуальные силовые тренажеры с изменяемой нагрузкой на основе сервопривода (перспективный проект)
}

\author{
В.В. Епишева , Д.Г. Максаков ${ }^{6}$, К.В. Коровин \\ В.В. Эрлиха ${ }^{a}$ А.А. Петров ${ }^{a}$, Л.Н. Петрова ${ }^{a}$

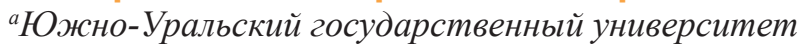 \\ (Национальный исследовательский университет) \\ Российская Федерация, Челябинск \\ ${ }^{6} О О О$ «Джимнес» \\ Российская Федерация, Челябинск
}

\begin{abstract}
Аннотация. Цель исследования - создание тренажера «нового поколения», функционал которого направлен на получение объективной информации (цифрового контроля) о выполняемой нагрузке, реакциях сердечно-сосудистой системы и опорно-двигательного аппарата во время физических упражнений. Был проведен патентный поиск, анализ публикаций, маркетинговые исследования и три научно-исследовательские опытно-конструкторские работы. Были сформированы технические требования к «интеллектуальным» силовым тренажерам. На базе тренажера «Гравитрон» созданы два прототипа, в которых грузоблочная система заменена на систему, состоящую из сервопривода и компонентов системы управления. Для контроля биомеханики движения был разработан прототип стабилометрической платформы, интегрированной в сиденье тренажера. Реализована функция биологической обратной связи, осуществляемая при помощи вывода на экран данных о положении центра давления в исходном положении и во время выполнения упражнений. Был создан прототип системы ЭКГ-мониторинга в I стандартном отведении, представляющий собой программно-аппаратный комплекс, встроенный в гриф силового тренажера. Первичные результаты свидетельствуют о технической возможности создания нового класса тренажерных устройств, обладающих функцией биологической обратной связи и функцией постоянного мониторинга состояния пользователя. Объединение нескольких интеллектуальных тренажеров в экосистему тренажерного зала позволит разработать принципиально новые подходы к тренировочному процессу.
\end{abstract}

Ключевые слова: силовой тренажер, сервопривод, мониторинг ЭКГ, контроль центра давления, интеллектуальный тренажер.

Работа выполнена в рамках госзадания Министерства науки и высшего образования РФ FENU-2020-0022 (№ 2020072ГЗ).

Научная специальность: 13.00.04 - теория и методика физического воспитания, спортивной тренировки, оздоровительной и адаптивной физической культуры. 


\section{Введение}

В «дорожной карте» национальных проектов «Демография» и «Здравоохранение», а также федерального проекта «Спорт - норма жизни» отведена большая роль привлечению населения к регулярным занятиям физической культурой и спортом (Passport of the Federal Project..., 2019). Для решения поставленной задачи необходимо разработать принципиально новые подходы к тренировочному процессу, создать тренажеры «нового поколения». Современные силовые тренажеры фактически только задают нагрузку и условную траекторию движения, степень которой определяется лишь по субъективным критериям.

Развитие спортивного рынка спорта и фитнеса в мире будет происходить в сторону постепенного увеличения сбора аналитических данных за счет накопления статистического материала, алгоритмов моделирования индивидуальных заключений и системы команд, направленных на коррекцию тренировочных процессов. Это, в свою очередь, будет стимулировать развитие индустрии технологий онлайнтренингов, в которых нагрузка будет стандартизированной, с учетом индивидуальной реакции организма человека. Система будет глобальной, и ее распространение будет происходить путем внедрения единых стандартов, протоколов и унификации платформ для подключения к ним устройств для сбора информации, с одной стороны, и выдачи тренировочных рекомендаций - с другой.

В 2019 году стартовал перспективный проект «Силовые тренажеры нового поколения», участниками которого являются ученые Института спорта, туризма и сервиса ЮУрГУ и индустриальные предприятия - ООО «Джимнес» и ООО «Велитек». Реализация проекта направлена на создание тренажеров нового поколения с применением технологий «персонального спорта». В тренажерах нагрузка будет регулироваться сервоприводом, работа которого будет управляться интеллектуальной системой с биологически обратной связью, анализирующей в реальном времени дан- ные выполняемой работы, ЭКГ и положения центра давления человека (стоя/сидя). Оценка техники выполнения упражнений, анализ реакции сердечно-сосудистой системы на нагрузку позволят системе строго индивидуализировать тренировочный процесс, рекомендовать последовательность выполнения, время восстановления между подходами, а также проводить сравнительный анализ эффективности занятий.

Цель исследования - создание силового тренажера «нового поколения», функционал которого направлен на получение объективной информации (цифрового контроля) о нагрузке, реакциях сердечно-сосудистой системы и опорнодвигательного аппарата во время выполнения физических упражнений.

\section{Материалы и методы}

С использованием баз данных Scopus, Web of Science и Google Scholar нами осуществлен патентный поиск и анализ публикаций по теме исследования. Проведены маркетинговые исследования и три научно-исследовательских опытноконструкторских работы: «Разработка опытного исследовательского стенда силового тренажера по типу «Гравитрон», «Разработка стабилометрической платформы для интеграции в конструкцию силового тренажера», «Разработка унифицированного модуля съема ЭКГ в I стандартном отведении для интеграции в линейку тренажеров нового поколения».

\section{Результаты}

По данным IHS Technology, текущий рынок гаджетов оценивается в \$7,5 млрд и продолжает стремительно расти (World Markets Healthcare, 2020). Согласно исследованию IDTechEx географическая сегментация мировых лидеров по поставке гаджетов в области здравоохранения выглядит следующим образом: США - 47 \%, Европа - $25 \%$, Восточная Азия - $22 \%$, другие - 6 \%. В целом сегмент «Спорт и здоровье» в последние годы растет со скоростью 40-80\% в год в основном за счет индустрии технологий «персонального спорта» 
и спортивной медицины. Россия имеет высокие шансы занять существенную долю новых рынков спортивных товаров и услуг, а также рынков продукции в области укрепления и развития здоровья («здоровье для здоровых») благодаря долговременным масштабным инвестициям в технологии спорта высоких достижений (олимпийского спорта) и бурного развития ИТ-отрасли в стране. Согласно расчетам рабочей группы в 2018 году глобальный объем сегмента «Спорт и здоровье» достигнет \$55 млрд в 2025 - \$241 млрд в 2035 - \$391 млрд. Объем российских товаров и услуг спортивного здоровья в 2016 году составит \$3,66 млрд в 2020 - \$22,78 млрд в 2035 - \$39,7 млрд. Предполагается, что доля российского сегмента от глобального сегмента «Спорт и здоровье» рынка «Хелснет» будет равна не менее $10 \%$ в 2035 году (Plan meropriyatij «Helsnet», 2016).

Целевыми сегментами потребителей создаваемого продукта были определены фитнес-организации и спортивные организации (в том числе профессионального сектора).

Разработаны требования к необходимым для реализации в тренажерах «нового поколения» технологическим решениям, определяющие его уникальность:

1. Алгоритм управления сервоприводом тренажера для дозирования нагрузки с шагом 0,5 кг в секунду и контролем количества повторений в режиме реального времени.

Научный поиск в Google Scholar по запросам «силовой тренажер-сервопривод», «тренажер-сервопривод» не выявил ни одной научной публикации, в т. ч. патентов. По запросам «Gym equipment servo», «strength machine servo» найдено несколько патентов:

- US5435798A Exercise apparatus with electronically variable resistance (Habing, Habing, Goodman, 1995).

- US4358105A Programmed exerciser apparatus and method (Sweeney Jr, 1982).

- US20120053014A1 Apparatus and System for a Resistance Training System (Zhu, 2012).
- US4865315A Dedicated microprocessor controlled exercise resistance machine $(\mathrm{Pa}-$ terson, DuPont, 1989).

- US5346452A Adjustable air resistance system for fitness equipment $(\mathrm{Ku}, 1994)$.

- US4678184A Constant force exercise device (Neiger et al., 1987).

- US6447431B1 Weightlifting device and method (Milburn, Courchesne, Andreatta, 2002).

- US5015926A Electronically controlled force application mechanism for exercise machines (Casler, 1991).

Bce указанные патенты схожи по направленности, но имеют принципиальные различия в конструкции и схеме управления, срок действия патентов истек. Следовательно, предлагаемый алгоритм и разработанный испытательный стенд в настоящее время являются инновационным продуктом, обладающим научной новизной.

Ближайшими конкурентами на рынке являются тренажеры HUR и Milon, в которых нагрузка в одном случае регулируется пневматической системой, в другом электронным сопротивлением (технология не раскрывается, предположительно электромагнит). Разработанный нами принцип дозирования нагрузки и алгоритм управления обладают значительно большим функционалом и возможностью интеграции в существующие тренажеры.

2. Алгоритм контроля положения центра давления человека в положении стоя и сидя с помощью тензометрических ковриков (платформ), позволяющих анализировать положение стоп, положение центра давления как в исходном положении, так и при выполнении упражнений.

Разрабатываемый алгоритм базируется на исследованиях постурального баланса (posture balance) и контроля за биомеханикой движения человека при помощи стабилометрической платформы (force platform), а также стелек с датчиками давления (smart insole). Главным принципом алгоритма является контроль за положением общего центра давления человека (center of pressure) во время выполнения упражнений с функцией обратной связи. 
3. Устройство контроля ЭКГ. Представляет собой интегрированные в гриф/ ручки тренажеров датчики ЭКГ (второе стандартное отведение) и расположенную в основании тренажера микросхему, позволяющие в реальном времени фиксировать частоту сердечных сокращений и ритм сердца.

Научный поиск в Google Scholar по запросу «strength machine with ECG measurement» (с учетом перестановки ключевых слов) не выявил ни одной научной публикации, в т. ч. патентов. В настоящее время на рынке не представлено силовых тренажеров с функцией регистрации ЭКГ в реальном времени.

4. Алгоритм биологической обратной связи. С помощью монитора, находящегося на уровне глаз выполняющего упражнения человека, будет отображаться информация о нагрузке, числе повторений, ЧСС, положении центра давления. Система перед выполнением упражнений будет рекомендовать занять правильное исходное положение (центр давления не смещен вправо/ влево, назад/вперед) и контролировать его смещение, динамику ЧСС.

5. Алгоритмы искусственного интеллекта, которые «ведут» тренировку с клиентом, выбирают программу и уровень интенсивности, следят за изменениями и дают обратную связь через монитор.

6. Алгоритмы управления последовательностью выполнения упражнений на разных тренажерах по типу «электронной очереди». На каждом тренажере будет установлено информационное табло, на котором исходя из рекомендованной методики тренировок будет загораться номер очередного клиента.

Предлагаемые технологические решения позволят осуществлять сбор и анализ данных, в т. ч. неструктурированной информации, которая будет использоваться для определения индивидуально-оптимальной программы тренировок. Уникальность собираемой информации даст возможность выявить наличие неочевидных, «скрытых закономерностей» определяющих эффективность тренировочного процесса.
Была проведена НИОКР «Разработка опытного исследовательского стенда силового тренажера по типу «Гравитрон» (Maksakov, 2019) и созданы два прототипа, в которых грузоблочная система заменена на систему, состоящую из сервопривода и компонентов системы управления (рис. 1). Характеристика сервопривода и редуктора:

- редуктор соосно-цилиндрический производства Varvel. Передаточное отношение 31,5; выходной момент 95,3 Нм;

- электродвигатель 0,75 кВт; номинальная частота вращения 3000 об/мин. Номинальный момент 2,39 Нм.

Тренажер «Гравитрон» был взят за основу ввиду того, что он может применяться как в составе спортивного зала, так и индивидуально в домашних условиях. Помимо интеллектуального наполнения, тренажер отличается компактностью, сопоставимой со шведской стенкой.

В настоящее время на его базе отрабатываются все алгоритмы управления и приводов для будущих тренажеров, в том числе:

1) единая экосистема тренажерного зала. Зал (тренажер) имеет систему распознавания - лица, отпечатка пальца, браслета, смартфона и т. д. (в зависимости от выбранных решений). Тренирующийся устанавливает у себя в телефоне приложение, в котором отражается его индивидуальный план тренировок. Все клиенты имеют приложение на телефоне, которое синхронизируется с каждым тренажером и автоматически устанавливает нагрузку;

2) система «страховки». Если во время выполнения упражнения человек не может выполнить тренировочный подход, то система управления постепенно снизит нагрузку для того, чтобы пользователь смог закончить упражнение.

В результате проведения НИОКР по теме «Разработка стабилометрической платформы для интеграции в конструкцию силового тренажера» разработана аппаратная часть и программное обеспечение системы управления нижнего уровня. В частности, для контроля движения применен 

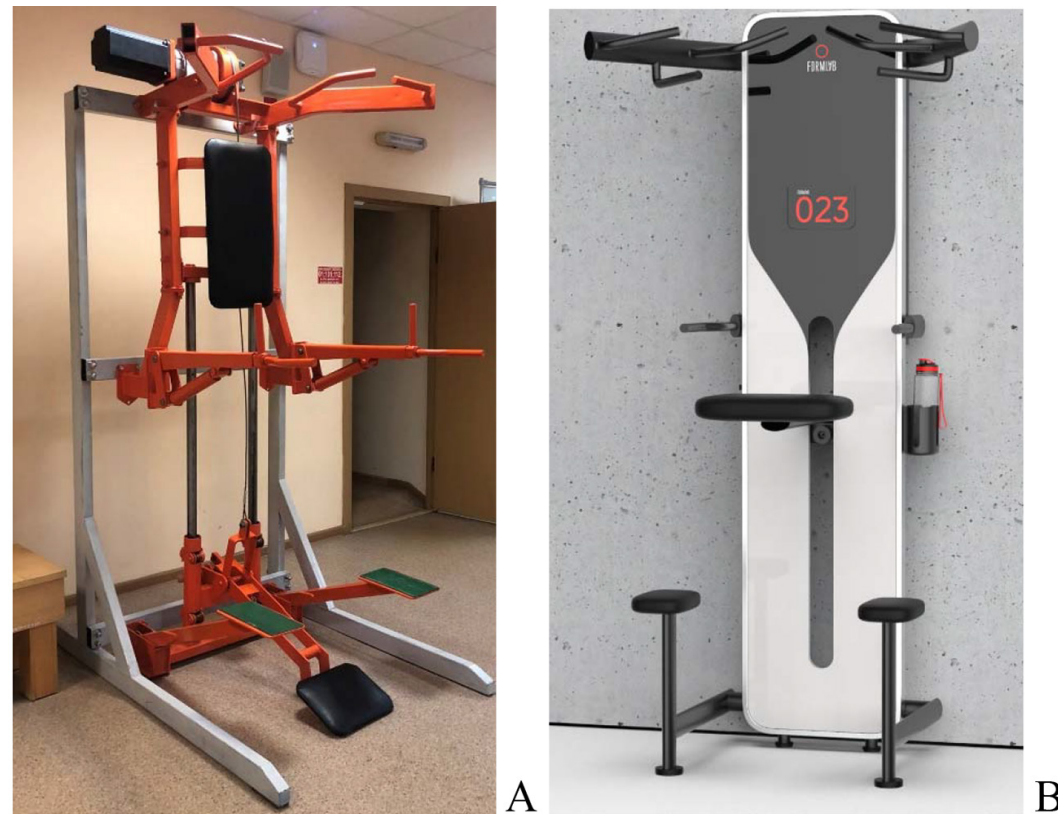

Рис. 1. Прототипы тренажера «Гравитрон» с сервоприводной системой нагружения: A - прототип № 1; B - прототип № 2

Fig. 1. Prototypes of «Gravitron» exercise machines with a servo-driven load system: A - prototype No. 1; B - prototype No. 2

принцип стабилометрической платформы, интегрированной в сиденье тренажера (рис. 2).

Стабилометрическая платформа представляет собой программно-аппаратный комплекс, предназначенный для определения центра давления во время выполнения упражнений и состоящий:

- из четырех тензодатчиков, расположенных в углах силовой рамы и жестко связанных с рамой тренажера;

- микроконтроллерного блока, включающего в себя аналогово-цифровые преобразователи, необходимые для функционирования тензодатчиков, а также 8-битный микроконтроллер;

- программного обеспечения микроконтроллера нижнего уровня, осуществляющего сбор данных с тензодатчиков, их преобразование, фильтрацию, применение первичных алгоритмов обработки и отправку в устройство верхнего уровня;

- планшетного компьютера с установленным специализированным ПО, ко- торое используется для сбора данных сс стабилометрической платформы, реализации биологической обратной связи, а также отправки данных в веб-приложение.

Характеристики платформы:

- масса тела спортсмена: от 40 до 120 кг;

- частота измерения: до 10 раз в секунду.

В результате работы стабилометрической платформы тренирующийся может наблюдать отклонение центра давления во фронтальной и сагиттальной плоскостях, что позволяет занять «правильное» исходное положение перед выполнением упражнения и контролировать технику выполнения упражнения (механизм биологической обратной связи).

В результате проведения НИОКР по теме «Разработка унифицированного модуля съема ЭКГ в I стандартном отведении, предназначенного для интеграции в линейку тренажеров нового поколения» были сформированы требования к аппарат- 


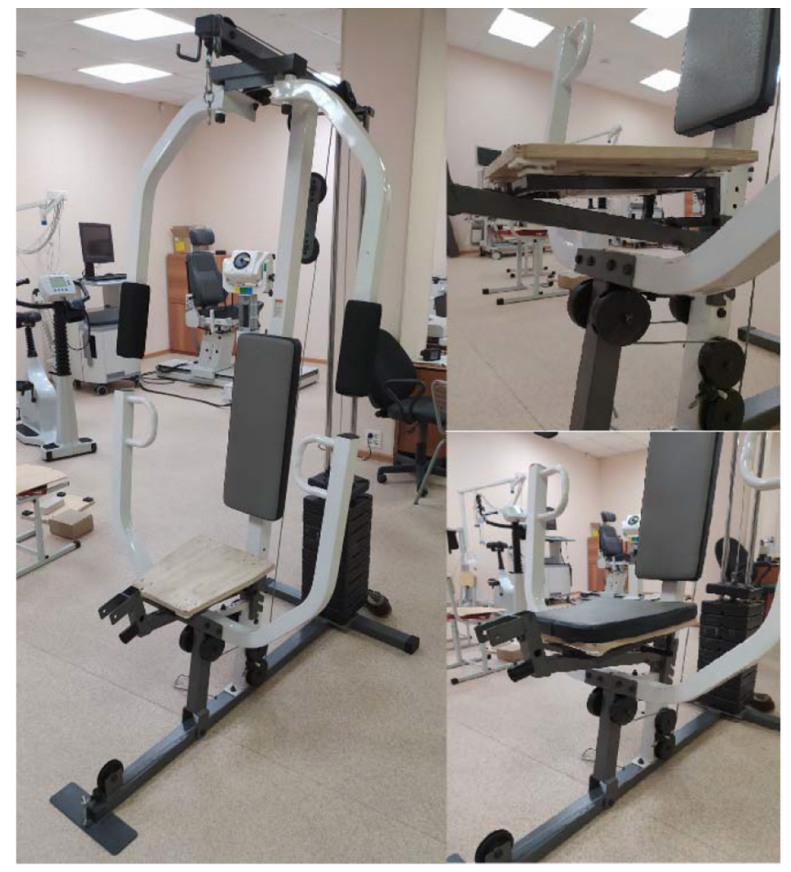

Рис. 2. Прототип стабилометрической платформы, интегрированной в конструкцию силового тренажера

Fig. 2. A prototype of a stabilometric platform integrated into the weight machine structure

ной части и осуществлена частичная сборка и испытания.

Модуль съема ЭКГ представляет собой программно-аппаратный комплекс, интегрированный в гриф тренажера и состоящий (рис. 3):

1) из модуля съема ЭКГ;

2) акселерометра с барометром;

3) микроконтроллерного блока на базе Arduino Nano c WiFi;

4) модуля питания.

На макетной плате были проведены первичные испытания устройства (рис. 4), показавшие устойчивую работоспособность и заявленную функциональность устройства. Применение акселерометра c барометром с функцией определения углов позволило дополнить устройство дополнительной функцией: определение асимметрии движения грифа и подсчет количества движений во время выполнения упражнения.

В дальнейшем в стандартный гриф верхней тяги силового тренажера были интегри- рованы датчики регистрации ЧСС и все модули аппаратной части устройства (рис. 5).

Характеристики устройства съема ЭКГ:

- гриф диаметром 20 мм;

- постоянный мониторинг ЧСС, ЭКГ в I стандартном отведении;

- частота измерения ЭКГ: до 100 раз в секунду;

- определение количества подъемов/ опусканий грифа, асимметрий в положении грифа относительно горизонтальной плоскости не менее 3 градусов;

- частота опроса акселерометра и барометра: до 100 раз в секунду;

- время автономной работы до 36 ч.

\section{Заключение}

Описанный перспективный проект НИР и НИОКР направлен на создание нового класса тренажерных устройств, обладающих функцией биологической обратной связи и постоянным мониторингом состояния пользователя. Наше представле- 


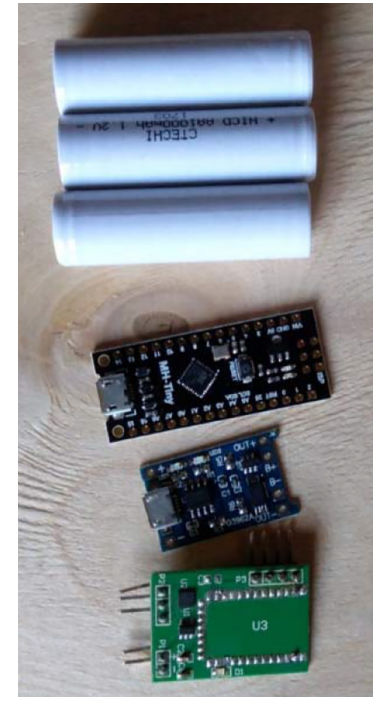

Рис. 3. Аппаратная часть модуля съема ЭКГ в I стандартном отведении

Fig. 3. Hardware component of the I standard lead ECG scoring system

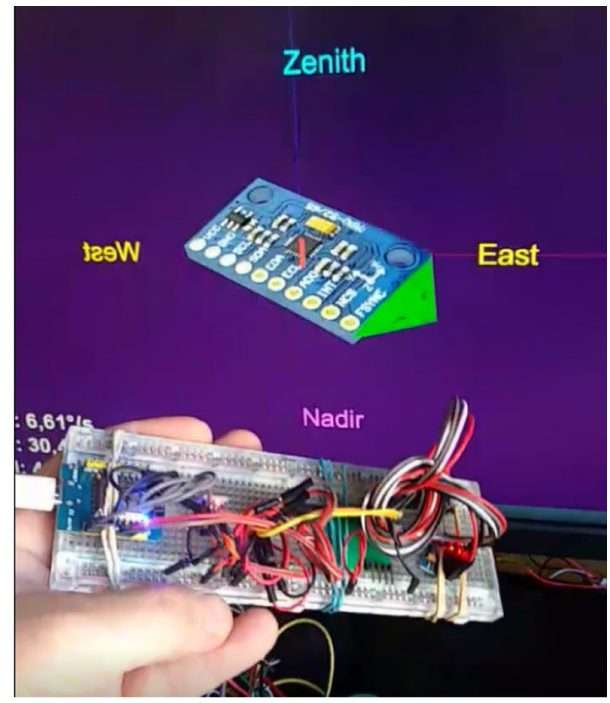

Рис. 4. Пример функциональных испытаний модулей на макетной плате

Fig. 4. An example of modules functional tests on a prototype board

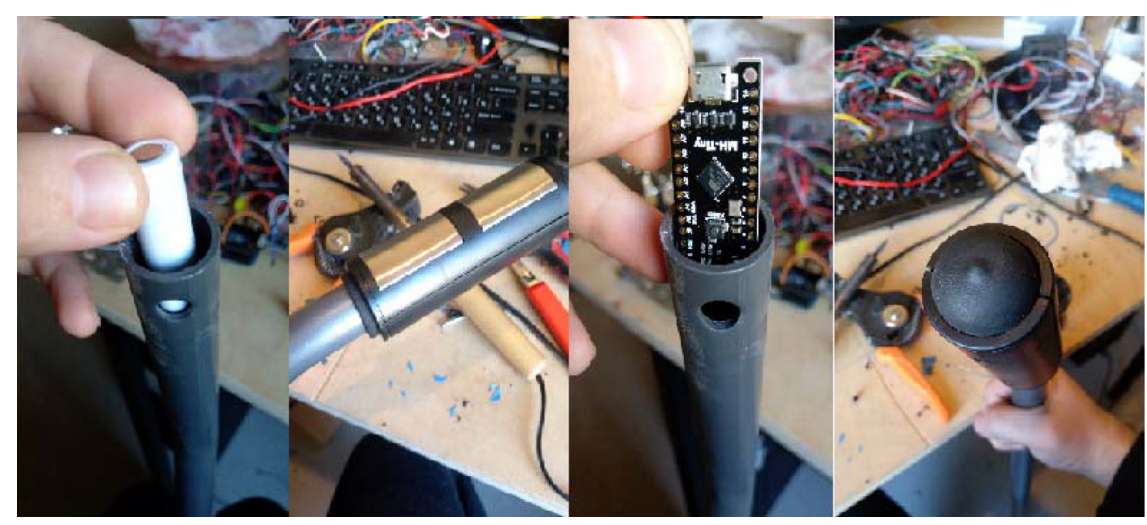

Рис. 5. Процесс интеграции аппаратной части в стандартный гриф силового тренажера

Fig. 5. The process of integrating hardware component into a standard weight machine bar

ние о «тренажерах будущего» базируется на следующих возможностях интеллектуального спортзала:

1. Перед началом тренировочного процесса с человека снимаются его функциональные возможности на данный момент (тесты физической подготовленности, анализ состава тела, ЧСС/ЭКГ в покое и т. п.). Производится анализ данных и выдаются рекомендации по тренировочному процессу в зависимости от поставленных целей.
2. Тренирующийся устанавливает у себя в телефоне приложение, в котором отражается его индивидуальный план тренировок. В будущем в процессе тренировок он наблюдает динамику показателей тренированности, а также может видеть выбранные группы друзей, с которыми идет соревновательный процесс в достижениях поставленных целей.

3. Перед началом упражнения на дисплее тренажера высвечивается информа- 
ция, где могут быть отражены предыдущие достигнутые показатели в этом упражнении, правильность выполнения, количество подходов на сегодня, весь цикл на данном тренажере. Программа на основе значений ЧСС определяет и подсказывает, когда необходимо закончить упражнение, дает команду к новому подходу/другому упражнению, если определяет, что сердечный ритм восстановился. Тренажер в момент может из- менять нагрузку, если констатирует, что человек не справляется с заданной нагрузкой или, наоборот, нагрузка недостаточна.

4. После окончания тренировочного процесса все данные автоматически загружаются на сервер для хранения и анализа, выдачи рекомендаций. Данные отправляются на учетную запись пользователя и отображаются в веб-приложении или в приложении на смартфоне.

\section{Список литературы / References}

Casler, J.A. (1991). U.S. Patent No. 5,015,926. Washington, DC: U.S. Patent and Trademark Office.

Draft Strategy 2030. The Development Strategy of Physical Education and Sports in the Russian Federation Until 2030. Available at: https://www.minsport.gov.ru/activities/proekt-strategii-2030/

Habing, T.G., Habing, D.J., \& Goodman, H. (1995). U.S. Patent No. 5,435,798. Washington, DC: U.S. Patent and Trademark Office.

Ku, T F. (1994). U.S. Patent No. 5,346,452. Washington, DC: U.S. Patent and Trademark Office.

Milburn, S.D., Courchesne, M.J., \& Andreatta, T.J. (2002). U.S. Patent No. 6,447,431. Washington, DC: U.S. Patent and Trademark Office.

Neiger, H.J., Genot, C.M., Prud'Hon, G.C., \& Schoens, M. (1987). U.S. Patent No. 4,678,184. Washington, DC: U.S. Patent and Trademark Office.

Passport of the Federal Project Creating Conditions for All Categories and Population Groups to Engage in Physical Education and Sports, Mass Sports, Including Increasing the Level of Provision of the Population with Sports Facilities and Preparing a Sports Reserve (Sport is the Norm of Life). Available at: https://rosmintrud.ru/ministry/programms/demography/5

Patent RU 193289 U1 Maksakov, D.G. (2019). Sportivnyj trenazher.

Paterson, D.F., \& DuPont, M. (1989). U.S. Patent No. 4,865,315. Washington, DC: U.S. Patent and Trademark Office.

PLAN meropriyatij («dorozhnaya karta») «Helsnet» Nacional'noj tekhnologicheskoj iniciativy. Available at: https://nti2035.ru/markets/docs/DK_healthnet.pdf

Sweeney Jr, J.S. (1982). U.S. Patent No. 4,358,105. Washington, DC: U.S. Patent and Trademark Office.

World Markets Healthcare. Available at: https://ihsmarkit.com/products/world-healthcare-markets. html

Zhu, Z. (2012). U.S. Patent Application No. 12/874,130. 